\title{
EVOLUCIÓN DEL E-GOVERNMENT EN COLOMBIA: el caso del Departamento del Atlántico
}

\section{EVOLUTION OF E-GOVERNMENT IN COLOMBIA: Departamento del Atlantico, a case study}

\begin{abstract}
RESUMEN
El presente estudio es una revisión bibliográfica de la evolución del E-government en Colombia tal como se ha aplicado en el departamento del Atlántico. Se concluye que, en Colombia, se ha otorgado importancia en los documentos oficialesa la infra e infoestructura; sin embargo, la revisión de los procesos en el departamento del Atlántico muestra que no se ha tenido en cuenta que estas necesitan de una actualización permanente y se evidencia que en materia de infoculturaaún hay mucho trabajo por hacer en el marco de apropiación de las TIC por parte de los ciudadanos, aunque los planes de desarrollo prioricen la necesidad de trabajar en esta área. El Estado colombiano es consciente de que el E-government es la alternativa más coherente al momento de optimizar los procesos de comunicación entre los gobiernos y los ciudadanosa través del establecimiento de plataformas amigables. Sin embargo, la revisión documental muestra que los esfuerzos han sido desarticulados y que existen falencias en los procesos de comunicación.
\end{abstract}

Palabras-clave: E-government. Infoestructura. Infocultura. Procesos de comunicación.

\begin{abstract}
The present study is a bibliographic review of the evolution of E-government as it has been applied in the departamento del Atlántico. The study concludes that in Colombia, official documents show the importance given to the infra and the infostructure. However, the revision of the processes in departamento del Atlántico, reveals the lack of investment in modernization and maintenance, and it is evident that in relation to infoculture there is a lot of work to do. Even though development plans give importance to infoculture, citizens have not had opportunities for social appropriation of TIC. The Colombian government is conscient that E-government is the most coherent alternative when seeking to optimize communication processes between governments and citizens through the establishment of friendly platforms. However, the review shows that efforts lack articulation and that there are many flaws in the communication processes.
\end{abstract}

Keywords: E-government. Info-structure. Info-culture. communication processes.

\section{INTRODUCIÓN}

1 Mestre em Coaching corporativo na EUROINNOVA, Espanha. Coordenadora de area organizacional, Faculdade de Humanidades. Universidad de la Costa, Colômbia. ORCID https://orcid.org/0000-0003-2758-9911. E-mail: marguiquintero@hotmail.com

2 Docente investigadora, Departamento de Comunicação Social. Coordenadora do Doutorado em Comunicação. Universidad del Norte, Colômbia. ORCID https://orcid.org/0000-0003-2812-9212.E-mail:paflores@uninorte.edu.co 
El hombre, desde el inicio de la historia, ha dado gran importancia a los procesos de comunicación y de relación con sus iguales. Esto ha traído como consecuencia la consolidación de un sistema social basado en las comunidades, las cuales dependen de cómo se dan las diversas relaciones al interior de ellas (Peters, 2017). Debido a la importancia que tienen la conexión y la comunicación entre los seres humanos, los gobiernos siempre se han preocupado por desarrollar la capacidad de enviar mensajes a la sociedad. Aún las monarquías absolutas buscaron recursos de comunicación para crear conexión entre el rey y sus súbditos al punto de que, como cuenta Peter Burke "ya en 1912, la gloriosa empresa de Luis XIV le recordaba a un estudioso francés la publicidad contemporánea" (1992, 2003, 13). Las democracias aumentaron esta necesidad de comunicación y ayudadas primero por el cine y la radio y luego, por la televisión crearon infinidad de recursos comunicativos para llegar a los ciudadanos. En las últimas décadas, las TIC han aumentado la posibilidad de comunicación entre los gobiernos y la ciudadanía debido a que ofrecen una interacción que las anteriores tecnologías no ofrecían. Es así cómo se han desarrollado estrategias para difundir las normas y procesos gubernamentales y los medios web se han convertido en aliados de la relación entre el gobierno y los ciudadanos (Strielkowski, Gryshova, \& Kalyugina, 2017; Rubio, Méndez \& Valencia, 2018).

Tal como lo plantean Soto y Miró (2016), en el mundo cibernético, para que estas normas de convivencia y entendimiento lleguen de mejor forma al público, es necesario garantizar la usabilidad, entendida como el grado de facilidad y comprensión en el uso de un tipo de producto digital: plataforma, website y sistema operativo, entre otros, de modo que satisfagan las necesidades de navegación al proporcionar la información buscada y responder a los estándares de navegación y de utilidad para quien ingresa.

La importancia de la usabilidad radica en que los clientes/usuarios visiten, aprovechen y regresen nuevamente a un sitio, generando mayor visibilidad, reduciendo tiempo y costos, proporcionando transparencia en los procesos y divulgación de la información, y agilizando la distribución y manejo de la información de manera masivay paralela a un número significativo de usuarios (Nielsen, 2000).

Desde el punto de vista histórico, se destaca que la implementación comercial de Internet, creada en 1969, generó nuevas dinámicas para realizar negocios al eliminar la distancia y presencialidad (Moon, 2002), transicionar de modelos de seguridad presencial a seguridad en línea, unificando el lenguaje y reorientando relaciones que eran entre empresa y consumidor hacia relaciones entre negocios (Muzzelec, Ronteau, Lambkin, 2015; Mittal, 2015). Internet no es una moda: se convirtió en una infraestructura críticapara los ecosistemas de negocios (Clearly \& Banasiewicz, 2018). 
Los cambios antes descritos fueron más allá del ámbito comercial, irrumpieron progresivamente en la cultura participativa y en la gestión pública, y los nuevos medios se convirtieron en un puente entre el gobierno y los ciudadanos. Surgen las llamadas Tecnologías para el Empoderamiento y la Participación (TEP), concebidas por Reig (2012) como un tipo de apropiación de las TIC capaz de propiciar una democracia electrónica o ciberdemocracia más evolucionada.

En definitiva, el uso de las TIC recreó y consolidó el concepto de Gobierno Electrónico en coherencia con la labor esencial del gobierno, lo que facilitaría la modernización del Estado reforzando la democracia en el sigloXXI (Cendrós, Durante \& Fermín, 2004), con base en la confianza, la corresponsabilidad y el compromiso cívico como elementos catalizadores (Ramírez, 2014), donde la co-creación, el co-diseño yla co-gestión de servicios públicos son elementos clave para el proceso de cambio (Ramírez, 2014).

En América Latina, el avance de Colombia, a cargo del Ministerio de Tecnologías de la Información y las Comunicaciones (MinTIC), se destaca por la realización de un arduo proceso de implementación del gobierno electrónico basado en la adquisición de infraestructura (Peña, Tarazona, \& Cuarta, 2017; Carreño, Bermúdez \& Rojas, 2018). Tras varios obstáculos en la implementación, en 2010, MinTIC modifica el modelo de implementación establecido hasta ese momento de Gobierno en Línea y genera un plan piloto para los departamentos de Atlántico, Antioquia y Casanare (Pozo, 2011).

En el marcosociopolítico, Colombia está constituida por 6 regiones: Insular, Caribe, Andina, Pacífica, Orinoquía y Amazonía, en las cuales hay 32 departamentos. En esta segmentación se destacan 5 ciudades principales por su número poblacional, actividad económica y aporte al PIB del país: Bogotá, Medellín, Cali, Barranquilla y Cartagena de Indias, respectivamente. Los tres departamentos seleccionados representan 3 de las regiones, Caribe (Atlántico), Andina (Antioquia) y Orinoquia (Casanare). El departamento del Atlántico se encuentra al norte del país, es el departamento más pequeño con una superficie de 3.388 km², 23 municipios y una población de 2.535.5171 personas, de las cuales el 94.84\% se ubican en las cabeceras urbanas, y solo $5.16 \%$ en la zona rural (Noguera, 2020).

La estrategia piloto en 2011 permitió destinar recursos para infraestructura, software, personal y acompañamiento del Estado. El resultado fue exitoso en cuanto a los indicadores técnicos lo cual hizo del Atlántico, el departamento pionero en infraestructura; sin embargo, la gestión del gobierno electrónico sigue siendo un reto en la actualidad, a pesar de que los gobiernos en turno la han establecido como un tema fundamental en sus Planes de Desarrollo. El abandono de la tecnología ha hecho, además, que la infraestructura que en un momento fue pionera, esté hoy desactualizada y se verifique una obsolescencia tecnológica de los sistemas. A partir del anterior recuento, el presente 
artículo se formula como una revisión bibliográfica de la evolución del E-government en Colombia y su aplicación en el contexto del departamento del Atlántico, de manera que se genere un análisis reflexivo sobre la temática abordada. El análisis consta de dos partes: E-government como herramienta de gobierno y E-government en Colombia y el Atlántico, logros y debilidades.

\section{METODOLOGÍA}

La metodología aplicada fue documental, pues permite la realización de un análisis de diversas fuentes bibliográficas y hemerográficas a través de un proceso sistemático de recolección, organización e interpretación para lograr identificar los datos provenientes de estas fuentes de información (Gómez, 2011). Estas fuentes se conformaron de la siguiente manera:

Tabla 1 - Fuentes de información

\begin{tabular}{|c|c|c|}
\hline $\begin{array}{r}\text { Documentos Oficiales } \\
\text { (Disponibles en la web) }\end{array}$ & $\begin{array}{c}\text { Fuentes Bibliográficas } \\
\text { (Digitales eimpresas) }\end{array}$ & $\begin{array}{c}\text { Fuentes Hemerográficas } \\
\text { (Digitales e impresas) }\end{array}$ \\
\hline 8 & 11 & 17 \\
\hline $\begin{array}{c}\text { Total fuentes } \\
\text { consultadas: } 36\end{array}$ & & \\
\hline
\end{tabular}

Fuente: Elaboración Propia (2020)

Entre las fuentes consultadas, se hizo especial énfasis en documentos oficiales (en formato digital) de índole gubernamental y artículos científicos que abordan la temática del E-government tanto a nivel internacional como en el ámbito colombiano y departamental. El método de análisis de la información escogido fue el análisis de contenido, que permite la revisión y presentación de los resultados obtenidos en categorías con un alto grado de análisis reflexivo sobre la temática (Monje, 2011).

\section{ANÁLISIS DE LOS DOCUMENTOS}

\subsection{El E-government como herramienta de gobierno}

El término E-government es definido como el uso de las TICs, en particular de Internet, para la gestión, planificación y administración de los gobiernos (Zúñiga et al, 2015) con el fin de ampliar los canales de democratización dónde todos tengan cabida con voz y voto (Yrivarren, 2017). Esto 
implica la reestructuración de los servicios públicos, un cambio cultural y organizacional, lo cual es un factor clave para eléxito de la instauración de un gobierno electrónico, sintraducirse únicamente en la automatización o instalación de software y hardware para los procesos de gestión (Rodríguez, 2011; Carreño, Bermúdez \& Rojas, 2018).

La relevancia del gobierno electrónico está en su capacidad de eliminar las distancias, agilizar los procesos, aumentar la eficiencia de la gestión pública e institucionalizar la transparencia en la administración, incrementando la capacidad de atención de los gobiernos locales, regionales y nacionales (Rodríguez, 2011).

Para visibilizar la implementación del E-government a nivel global, la ONU ha venido publicando desde 2003, un ranking bianual que tiene cuatro indicadores que establecen las posiciones de los 193 países afiliados a la ONU a través de 4 eslabones de medición los cuales son: a) Índice de Desarrollo de Gobierno Electrónico, b) Índice de servicio en línea, c) Índice de infraestructura de telecomunicaciones y d) Índice de capital humano, establecidos para asegurar la implementación de la E-democracy. Por otro lado, se destaca que, con el fin de generar estas nuevas dinámicas, el E-government o Gobierno electrónico tiene tres frentes de acción tal como describen Laskaridis et al (2008):-

- $\quad$ Gobierno a gobierno (G2G): busca el intercambio de datos electrónicos entre actores gubernamentales.

- Gobierno a empresa o Government to Business (G2B): el cual se centra en las transacciones de negocios.

- Gobierno a consumidor/ciudadano (G2C): las iniciativas para facilitar la interacción entre las personas y elgobierno como consumidores de servicios públicosy como ciudadanos.

A estos frentes, Náser y Concha (2011) añaden una cuarta relación, entre el gobierno y sus empleados G2E. Estosfrentes deacción están directamenterelacionadoscontresfactoresfundamentales del E-goverment definidos por Pimienta (2007), los cuales se visualizan en el siguiente gráfico: 
Figura 1 - Rumbos de la inversión en proyectos de TICpD

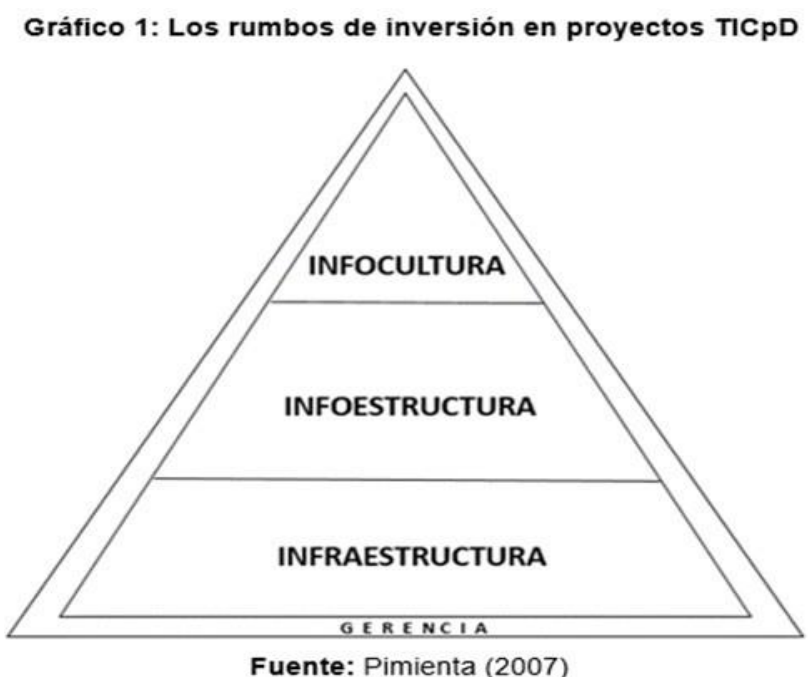

Fuente: Adaptado de Pimienta (2007)

Con base en el grafico anterior, se considera esencial la existencia de 3 factores: infraestructura, definida como los dispositivos que permiten la transmisión de la señal (microondas, satélites), el transporte (protocolos de comunicación) y dispositivos de computación (sistemas operativos); infoestructura, la cual hace referencia a los contenidos y aplicaciones de alojamiento (programas, bases de datos y website); y la infocultura, el componente de apropiación visto en el conocimiento, los métodos, las prácticas y las reglas de buen uso que poseen las personas que se han apropiado del manejo de la comunicación y de la información en red (Pimienta, 2007). El balance entre los factores es el garante del aprovechamiento de las TIC, el desarrollo humano desde la E-democracy y la permanente actualización de los software y hardware.

\subsection{El E-government en Colombia y en el Atlántico: Logros y debilidades}

Tal como se ha mencionado, la tendencia mundial toca suelo colombiano a raíz del boom de Internet como red global capaz de conectarnos a todos y como plataforma de transparencia por la publicación de información de las entidades pública en tiempo real.

En el año 2000, durante la presidencia de Andrés Pastrana, se estableció un marco de Gobierno Electrónico en el cual debían trabajar los ministerios, departamentos administrativos, superintendencias, unidades administrativas especiales, entidades descentralizadas del orden nacional, 
empresas industriales y comerciales del Estado y las sociedades de economía mixta, para la generación del "Gobierno Electrónico" (Massal \& Sandoval, 2010).

El objetivo delapolíticapresentadasecentró enlacapacidad de proveer alEstado delaconectividad que facilitara la gestión pública y los servicios al ciudadano como complemento del esquema del mundo globalizado e interconectado a través de los medios electrónicos (Presidencia de Colombia, 2000), lo que permitiría una implementación que intrínsecamente equilibraría la infraestructura, la infoestructura y la infocultura. En términos organizacionales, las entidades públicas en Colombia son reguladas a través de los llamados "Manuales" que explican la hoja de ruta, normativas e indicadores para la implementación de las políticas, en este caso de las relacionadas con el E-govermnent. Sin embargo, la revisión de los documentos muestra que están dirigidos al aseguramiento de la infraestructura y la infoestructura desequilibrando los rumbos de la inversión en proyectos de TICpD tras la desarticulación con la comunidad. Así, la transición de componentes ha sido la siguiente:

Tabla 2 - Comparación de componentes

\begin{tabular}{|l|l|}
\hline $\begin{array}{c}\text { Manual Estrategia de Gobierno en Línea } \\
(\mathbf{2 0 1 5 )}\end{array}$ & Manual de Gobierno Digital (2018) \\
\hline Componentes: & Componentes: \\
1. TIC para gobierno abierto & 1. TIC para el Estado \\
2. TIC para servicio & \\
3. TIC para la gestión & \\
4. Seguridad y privacidad de la \\
información
\end{tabular}

Fuente: Propia (2019)

La gestión pública es sustantivamente gestión, como conjunto de reglas y decisiones dirigidas a coordinar las acciones, y es pública porque persigue metas colectivas y se inscribe en las restricciones jurídico-políticas (Villanueva, 2015). En el mundo del E-govenment los componentes TIC se apropian del concepto de gestión y se entienden como las líneas de acción que orientan el desarrollo y la implementación de la política. Para lograr esto, se mejoran sus beneficios por la distribución del conocimiento, elemento base de la planeación estratégica organizacional fundada en sus stakeholders y son las TIC las que ofrecen plataformas de interés como un espacio para la cercanía (Moreno, 2010). 
En Colombia, el producto visible del E-government fue el portal www.gobiernoenlinea.gov.co, el cual se concentró en divulgar y facilitar el acceso de la información pública a los ciudadanos, funcionarios yotrasentidades estatales, al trasladar losservicios presenciales alas plataformas digitales.

En perspectiva, a partir de la "Agenda de Conectividad" en 2002, se publicó la Ley 790 de Renovación de la Administración Pública basada en el programa de Gobierno en Línea. De 2003 a 2006 las normativas se multiplicaron y se detallaron aún más los procesos. La ley 812 definió los programas prioritarios de la renovación de la Administración Pública: el uso de las TIC, el documento Conpes 3249 y 3292 establecieron políticas para la racionalización, normalización y sistematización de trámites (Pozo, 2011; Cardona, Cortés \& Ujueta, 2015) lo cual facilitó el camino para la infoestructura haciendo más sencillos la gestión y alojamiento del contenido.

En 2006 se aprueba el Decreto 2434 seguido de la Ley 1150 de 2007 que buscan la transparencia en lacontratación pública mediante eluso de las TIC; estas y las normas siguientes, se encuentran en el Manual de Política de Gobierno Digital publicado por MinTIC a finales de 2018 resultado de procesos de participación ciudadana.

\subsection{E-government en el departamento del Atlántico}

La dificultad para establecer el avance o retroceso en los procesos de implementación del gobierno electrónico en el mundo se debe, fundamentalmente, a la diversidad de modelos existentes, tal como lo establecen Siau y Long (2005). Debido a esto, los autores proponen, tras una revisión de los diversos modelos, una implementación en 5 fases ascendentes: Información, Interacción, Transacción, Transformación y Democracia.

En Colombia, mediante el Decreto 1151 de 2008, se establece este modelo de implementación por fases para lo cual se redacta y divulga el "Manual para la implementación de la Estrategia de Gobierno en Línea", la cual será de obligatorio cumplimiento para todas las entidades pertenecientes a la Agenda de Conectividad. La última fase, la de E-democracia- debía estar finalizada, a más tardar, el 1 de diciembre de 2012.

Para medir el progreso de la implementación, el Gobierno Nacional (MinTIC, 2011) crea cuatro niveles: Inicial, el cual determina si existen las condiciones para habilitar el componente; Básico donde se logra el entendimiento y la organización, Avanzado para indicar que ya está interiorizado y ofertando la mayoría de los servicios, por último, el Mejoramiento permanente que induce a la innovación. 
En el departamento del Atlántico, los diagnósticos y registros base para la fundamentación del plan de desarrollo Atlántico Más Social 2012-2015, permiten concluir que hasta 2011 el departamento había logrado llegar al 63\% de implementación de la tercera fase de Transacciones mediadas por la web (Segebre, 2012). Sin embargo, el nuevo plan de gobierno ejecutado entre 2012 y 2015, en su rendición de cuentas afirma haber avanzado un $17 \%$ adicional en cuatro años en la misma fase de transacciones, llegando así al $80 \%$ de una fase intermedia que aun demanda la transformación y democracia como objetivos últimos del E-government. Paralelo a la implementación, el Atlántico había dado diversos pasos enfocados a la apropiación del E-government a través de la creación del observatorio de las TIC en 2008, con modelos innovadores de producción mediante la tecnología y la promoción del teletrabajo en 2016 (Verano, 2008; Verano, 2016).

Pese a ello, existen grandes falencias relacionadas con los indicadores de sensibilización interna de los funcionarios o con la apropiación de las TIC por parte de la sociedad, (Verano, 2016) quienes deberían ser los stakeholderprincipales. Esto se debe a que existe una omisión de varios de los planteamientos del MinTIC con respecto a la gestión de las TIC (Chaparro \& Guerrero, 2017), lo que ocasiona altos niveles de resistencia al cambio, caracterizada por una falta de apropiación del elemento humano (Ospina, Tarazona \& Rodríguez, 2017).

Durante esta difícil etapa del confinamiento debido a la pandemia que exige el distanciamiento social, se ha hecho más visible la necesidad de proporcionar conectividad a las personas para garantizar la comunicación de los gobiernos con sus ciudadanos, además de dar continuidad a los procesos educativos, comerciales y laborales. Tanto el MinTIC como la Gobernación del Atlántico reconocen que en 2020 se deben priorizar 5 municipios con barreras tecnológicas: Campo De La Cruz, Galapa, Luruaco, Repelón, Santa Lucía junto a Puerto Colombia yel distrito de Barranquilla como Área Metropolitana (Ramo, 2020). Lacapital del departamento que, en 2011, había sido considerado pionera en la implementación del E-government, una década demanda una nueva reinversión millonaria y se reconoce su atraso en los procesos de gobierno en línea.

En la actualidad, el nuevo gobierno nacional tiene como meta llevar Internet a los hogares de estratos 1 y 2 de la ciudad con tarifas subsidiadas denominadas tarifa social. Por su parte, a nivel local el MinTIC busca realizar el piloto tecnológico para la implementación del 5G en Barranquilla, invitando así a los gobernantes locales a tener una mejor articulación y eliminar barreras para el despliegue de infraestructura (MinTIC, 2020). Este panorama desconoce la necesidad de la apropiación social o 
infocultura, la cual ha estado ausente y hoy demanda inversiones superiores a $\$ 2$ billones de pesos colombianos provenientes del recaudosocial.

La dualidad del E-Government en el Atlántico está en la priorización de la infoestructura e infraestructura frente a una necesidad latente de vincular a la comunidad mediante la infocultura. Si bien han existido algunas iniciativas de educación, éstas no pueden funcionar si los componentes técnicos no están adecuados y habilitados para la población. Una encuesta informal realizada en 12 de los municipios del Atlántico en el mes de noviembre de 2019, reveló que la conectividad en parques y plazas del municipio se había suspendido y que el $92 \%$ de los ciudadanos solicitaban la solución de esta situación ya que, debido a las condiciones socioeconómicas de la mayoría de la población, conectarse dependía de la disponibilidad de este servicio.

El anterior recuento demuestra que el equilibrio entre los componentes de los rumbos de la inversión en proyectos de TICpD planteado por Pimienta, (2007) es la estrategia viable para alcanzar la implementación total del E-govenment y masificar los beneficios entre los frentes de acción G2C, G2B, G2G Y G2E. Mientras no se atiendan de manera correcta la infoestructura e infraestructura siempre tendrá vacíos la infocultura.

\section{CONSIDERACIONES FINALES}

En concordancia con el crecimiento exponencial de las sociedades, se evidencia que el E-government surge como una herramienta para no perder la personalización y el relacionamiento directo con un Estado que debe trabajar para y en pro de sus ciudadanos. Así, el desafío se centra en el equilibrio de lo que Pimienta plantea como rumbos de inversión en proyectos TICpD lo que procura por un equilibrio entre infraestructura, infoestructura e infocultura, trasversales para las relaciones con los actores de la democracia.

Es así como se entiende que el E-government es una revolución a nivel de gobernanza, gestión pública y transparencia ya que permite la creación de espacios seguros, amigables y rápidos de atención al ciudadano a través del uso de las TIC, facilitando el acceso a los procesos y rompiendo con las brechas territoriales y sociales. Ello implica asegurar la apropiación tecnológica entre las generaciones.

Desde la perspectiva colombiana, se concluye que por casi tres décadas se vienen dando pasos significativos, logrando un avance relevante en la última década con las políticas de modernización del país agregadas, donde pese alos aciertos, desaciertos y factores por mejorar, se evidencia un mecanismo 
de evaluación permanente que ha replanteado desde los modelos de implementación, pasando por la normativa hasta la consideración por fases y entidades competentes al tema que generen una cultura en torno a las TIC, lo que exige un trabajo arduo de cambio de percepción de los ciudadanos frente a sus instituciones públicas.

A raíz de lo anterior en 2018, en busca de recolectar los intentos jurídicos anteriormente explicados desde 1991, el Ministerio de las Tecnologías y la Comunicación MinTIC creó el Manual de Gobierno Digital como una de las diecisiete políticas de gestión y desempeño institucional, que se desarrolla en el marco del Modelo Integrado de Planeación y Gestión y se encuentra en el Eje de Gestión para el Resultado con Valores. Esta política, según lo establecido en el Manual, establece la búsqueda de un aprovechamiento real de las TIC para suplir las necesidades de la población.

En el caso del departamento del Atlántico, en esta visión gubernamental no se constata específicamente la esencia del gobierno en línea bajo los intereses de la comunidad, en coherencia con la rectitud del buen obrar para el beneficio de un país que reclama, con justa causa, menos trámites, menos filas, menos papeleos, menos corrupción y más rapidez (Pozo, 2011).

Es así como esta revisión documental nos deja ver un desequilibrio de los rumbos de inversión en proyectos TICpD, pero un alto énfasis en la gestión de infraestructura e infoestructura, como lo deja ver en la rendición de cuentas de la gobernación del Atlántico al contar una zona wifi cada $15 \mathrm{Km}$, al proveer 137 zonas en los municipios y 100 en Barranquilla (Verano, 2019) Esto fue acompañado de una tímida propuesta de apoyo a la innovación digital más que al E-government, denominada Atlánticonnect, una apuesta al trabajo colaborativo público-privado con el fin de impulsar el emprendimiento para el desarrollo social y económico del departamento, lo cual no es suficiente para llegar a la E-democracy.

Aun se necesita mucha disposición política y despliegue de estrategias comunicativas para la implementación de la infocultura, contemplando los 8 pasos para gestionar la gestión del cambio o la resistencia al mismo: 1 Crear sentido de urgencia, 2 formar una coalición, 3 crear visión para el cambio, 4 comunicar la visión, 5 eliminar los obstáculos, 6 asegurar triunfos s corto plazo, 7 construir sobre el cambio; 8 acciones enfocadas a permear la forma de hacer las cosas en el ámbito público.

\section{REFERENCIAS}

ALBI, E.; GONZÁLEZ, J.; LÓPEZ, G. 1997. Gestión pública. Fundamentos técnicas y casos. Ariel Economía, 1997. 
ALLISON, G. Public and private management: Are they fundamentally alike in all unimportant respects? In new directions in public administration. Palo alto: Bozeman and J. Straussman, Mayfield Publishing. 1984.

BURKE, P. 2003. La fabricación de Luis XVI. Nerea: Madrid

CARDONA, D.; CORTÉS, J.; UJUETA, S. Gobierno Electrónico En Colombia: Marco Normativo y Desempeño de Tres Índices Estratégicos. Revista Venezolana de Gerencia, v. 19, n. 69, p. 11-34, 2015.

CARREÑO, J.; BERMÚDEZ, G.; ROJAS, L. Plataforma virtual como mecanismo de gobierno electrónico entre población joven y administración local de Mosquera, Colombia. Ingeniería solidaria, v. 14 , n. 24 , p. $1-12,2018$.

CENDRÓS, J.; DURANTE, C.; FERMÍN, J. Factores estratégicos para desarrollar el gobierno electrónico en las Alcaldías de Venezuela. Opción, v.20, n. 45, p. 98-114, 2004.

CLEARY,P.;BANASIEWICZ, A. Toward Resilience of Business Ecosystems: The Internet as a Critical Infrastructure. Australian Academy of Accounting and Finance Review, v. 4, n. 1, p. 1-10, 2018.

GÓMEZ, L. Un espacio para la investigación documental. Revista Vanguardia Psicológica Clínica Teórica y Práctica, v. 1, n. 2, p. 226-233, 2010.

LASKARIDIS, G.; MARKELLOS, K.; MARKELLOU, P.; PANAYIOTAKI, A. E-Government's Barriers and Opportunities. Handbook of Research on Public Information Technology, 2008.

MASSAL, J.;SANDOVAL, C. Gobierno electrónico. ¿Estado, ciudadanía y democracia en Internet? Análisis político, v. 23, n. 68, p. 3-25, 2010.

MINISTERIO DE LAS TIC. “Decreto 1151 de 2008”. Disponible en http://goo.gl/bCRHo. Consultado el 20.04.2019. 2008.

MINTIC. Este es un Gobierno que estábuscando llegar a todos los municipios del Atlántico con algún servicio TIC: ministra Constaín. 2020. Disponible en https://bit.ly/2YLDtve

MINTIC. Manual para implementar estrategia GEL3.0. 2011. Disponible en https://bit.ly/31FK7WQ

MITTALI, B. Familiarity, Leisure, Role Overload, Communication, and Opportunism: Uses and Misuses of the Internet. Academy of Marketing Science, 2015.

MONJE, C. Guía didáctica para la investigación cualitativa. Universidad Surcolombiana, 2011.

MOON, J. The evolution of e-government among municipalities: rhetoric or reality? Public Administration Review, v. 62, n. 4, p. 424-433, 2002.

MORENO, J. Procesos de gestión pública innovadora: el caso de los módulos de atención a migrantes en Baja California. Estudios Fronterizos, v. 11, n. 21, p. 143-170, 2010. 
MUZELLEC, L.; RONTEAU, S.; LAMBKIN, M. Two-sided Internet platforms: A business model lifecycle perspective. Industrial Marketing Management, v. 45, p. 139-150, 2015.

NACIONES UNIDAS. E-government Knowledgebase, 2003. Disponible en: https://publicadministration. un.org/egovkb/en-us/About/UNeGovDD-Framework

NASER, A., \& CONCHA, G. (2011). El gobierno electrónico en la gestión pública. Cepal. Disponible en https://bit.ly/2VKcEXM

NIELSEN, J. Usability 101: Introduction to usability. N/N Group, 2003 Disponible en http://didattica. uniroma2.it/assets/uploads/corsi/143228/Nielsen_5_articles.doc.

NIETO, W.; CHAPARRO, Y.; GUERRERO, J. Guía de buenas prácticas, instrumento para desarrollar la arquitectura de tecnología informática en la Gobernación del departamento del Atlántico, con la intención de habilitar la estrategia de TI para la gestión y el gobierno de la información. (Tesis de maestría). Universidad del Norte,2018.

NOGUERA, E. Plan de Desarrollo 2020-2023. Atlántico para la Gente, 2020.

NÚÑEZ, R.; PALACIOS, J.; PÉREZ, L. Correlación de la interacción de los ciudadanos y gobierno electrónico estatal. Pistas Educativas, v. 40, n. 131, 222-231, 2018.

PETERS, J. Hablar al aire: una historia de la idea de comunicación. Ciudad de México. Fondo de Cultura Económica, 2017

PIMIENTA, D. Brecha digital, brecha social, brecha paradigmática, 2007. Disponible en http:// funredes.org/mistica/castellano/ciberoteca/tematica/brecha_paradigmatica.pdf.

POZO, K. Retos digitales: A propósito de Gobierno en Línea en el Atlántico. (Tesis de maestría). Universidad del Norte, 2011

PASTRANA, A. Directiva Presidencial No. 02. Presidencia de Colombia Disponible en: https://www. mintic.gov.co/portal/604/articles-3646_documento.pdf.

RAMÍREZ, Á. Gobierno abierto. EUNOMíA. Revista en Cultura de la Legalidad, v. 1, p. 201-216, 2014.

REIG, D. Disonancia cognitiva y apropiación de las TIC. Revista TELOS, v. 90, n. 1, p. 9-10, 2012

RODRÍGUEZ, G. Gobierno Electrónico: Hacia la modernización y transferencia de la gestión pública. Revista de Derecho, v. 21, n. 21, p. 1-23, 2011.

ROYERO, J. Del e-gobierno a la e-universidad: una visión desde América Latina. Universities and Knowledge Society Journal, v. 4, n. 2, p. 1-15, 2007.

ROMO, N. Atlántico tiene 7 municipios con barreras tecnológicas: Mintic. El Heraldo. 2020. Disponible en https://bit.ly/38q7Ziw

SEGEBRE, J. Plan de Desarrollo 2012-2015. Atlántico Más Social, 2012. 
SOTO, A.; MIRÓ, J. Usabilidad y accesibilidad para un e-learning inclusivo. Revista de educación inclusiva, v. 2, n. 1, p. 49-60, 2016.

VERANO, E. Plan de Desarrollo Departamental 2008-2011. Por el Bien del Atlántico, 2008.

VERANO, E. Atlántico Líder. Plan de Desarrollo. Gobernación del Atlántico, 2016.

VERANO, E. Informe de evaluación a la audiencia pública de rendición de cuentas de la gobernación del atlántico-2018. Gobernación del Atlántico, 2019.

VILLANUEVA, L. Gobernanza y gestión pública. Fondo de cultura Económica, 2015.

YRIVARREN, J. Gobierno electrónico: Análisis de los conceptos de tecnología, comodidad y democracia. (Tesis). Universidad Peruana de Ciencias Aplicadas, 2017.

ZÚÑIGA, R., CASTILLO, O.; HERNÁNDEZ, E.; CERVANTES, G. Análisis general del gobierno electrónico en México. PAAKAT: Revista de Tecnología y Sociedad, v. 5, n. 9, p. 10-20, 2015. 